\title{
PERSISTENT HYPERTENSION ASSOCIATED WITH SLEEP DISORDERED BREATHING IN ADOLESCENCE: COMPREHENDING THE CAUSE AND THE EFFECT.
}

Brajesh Mishra1 ${ }^{1}$ Bhawesh Mishra².

1. Consultant Pulmonologist, Department of Pulmonology, Shree Jagannath Hospital and Research Centre, Ranchi.

2. Senior Resident, Department of Biochemistry, Lady Hardinge Medical College, New Delhi.

\section{CORRESPONDING AUTHOR}

Bhawesh Mishra,

Department of Biochemistry,

Lady Hardinge Medical College,

New Delhi.

Email-drbhawesh@gmail.com

\section{HOW TO CITE THIS ARTICLE:}

Brajesh Mishra, Bhawesh Mishra. "Persistent Hypertension Associated with Sleep Disordered Breathing in Adolescence: Comprehending the Cause and the Effect". Journal of Evolution of Medical and Dental Sciences 2013; Vol2, Issue 25, June 24; Page: 4559-4562.

ABSTRACT: Hypertension in childhood and adolescence is usually secondary to underlying causes like renovascular, renal-parenchymal, cardiovascular and endocrine diseases. Persistent hypertension in paediatric age group is a diagnostic and therapeutic challenge. Sleep disordered breathing or Obstructive sleep apnea in adolescence can present in numerous non- specific ways. Whether sleep disordered breathing can solely be a cause of persistent hypertension in adolescence is doubted. We report a case of a 13 year old boy with history of persistent hypertension inadequately controlled even with multiple antihypertensive drugs. A thorough history, physical examination and rational investigations were performed to rule out causes of secondary hypertension. The patient was obese and obstructive sleep apnea was identified on the basis of history and sleep studies. He was titrated on continuous positive airway pressure (CPAP) for obstructive sleep apnea and put on a weight loss regimen. Amelioration of symptoms and adequate blood pressure control was obtained. We concluded that hypertension in adolescence may be caused or aggravated by sleep disordered breathing and a specific treatment approach may help in relieving symptoms and achieving adequate blood pressure control especially when no other etiology or risk factors can be identified.

KEYWORDS: Obstructive sleep apnea, persistent hypertension, Sleep disordered breathing, adolescence, childhood obesity.

INTRODUCTION: Hypertension is not uncommon in childhood and adolescence. The usual causes are secondary, due to renovascular, renal-parenchymal, cardiovascular and endocrine diseases (1). Any evidence of persistent hypertension (HT) in paediatric age group is a diagnostic and therapeutic challenge especially when the cause is unusual. Sleep disordered breathing (SDB) and obstructive sleep apneas (OSA) are becoming increasingly common in paediatric age group possibly due to changes in lifestyle and food habits. OSA is characterized by prolonged partial upper airway 
obstruction, intermittent complete or partial obstruction (obstructive apnoea or hypopnoea) or both prolonged and intermittent obstruction that disrupt normal ventilation during sleep, normal sleep patterns or both (2). SDB in children and adolescents can present in numerous ways and often the signs are non- specific. Whether sleep disordered breathing in children can solely be a cause of persistent HT is a matter of debate (3).

CASE REPORT: A 13 year old boy presented in outpatient department of our super speciality hospital with history of chronic headaches and lethargy for more than six months. History revealed that he was diagnosed for HT, 2 years back. Since then, he was put on various antihypertensive drugs but his blood pressure(BP) was not adequately controlled even on combination antihypertensive therapy (Amlodipine $5 \mathrm{mg}+$ Atenolol $50 \mathrm{mg}+$ Hydrochlorothiazide 12.5mg) and reportedly remained persistently higher than $140 / 90 \mathrm{~mm}$ of $\mathrm{Hg}$. On examination patient was conscious, oriented, obese with weight $60 \mathrm{Kg}$, height $137 \mathrm{~cm}$ and $\mathrm{BMI}=32$. Pulse=68/min, regular, $\mathrm{BP}=$ $160 / 110$. Chest was bilaterally symmetrical and clear on auscultation. Heart sounds were normal and regular. Abdominal and neurological examinations were also normal. On oro-nasopharyngeal examination, no anatomical or neurological deformity was observed. There was no family history of HT reported. Routine blood biochemistry, including renal function tests were found normal. Urine tested negative for proteins, catecholamines and vanillylmandelic acid (VMA). The patient was further investigated for common causes of secondary HT for his age group. Renal arteriogram was normal and no evidence of renal parenchymal disorder was found. There was no history of drug intake except antihypertensives.

The patient was re-evaluated and a thorough history disclosed that he always complained of lack of freshness and persistent fatigue. His parents mentioned that they have received several complaints from his school about his sleeping in class and performing poorly. They also mentioned that he was a heavy snorer at night and no one from family wanted to sleep in his room. The patient was screened for OSA. Evaluation based on "Epworth sleepiness scale" yielded a score of 17(out of possible 24 points) affirming excessive daytime sleepiness. Full night sleep study revealed his apnea hypopnea index (AHI) was elevated at 22 events/hr (normal $<5$ events/hr). The patient was titrated on continuous positive airway pressure (CPAP) delivered via a nasal mask at $10 \mathrm{~cm} \mathrm{H}_{2} 0$. He was also put on an exercise and diet regime for weight loss.

Follow up after one month showed marked improvement in his daytime sleepiness. He also managed to lose $3 \mathrm{Kg}$ of weight. His antihypertensive medication was reduced to Amlodipine $2.5 \mathrm{mg}+$ Hydrochlorthiazide $12.5 \mathrm{mg}$ with BP controlled at $130 / 80 \mathrm{mmHg}$. Complaints of day time sleepiness had remarkably improved with decreased snoring at night. His performance at school also improved. Subsequent follow-ups showed further improvement in symptoms and BP under control on lower antihypertensive doses.

DISCUSSION: Persistent HT in children is defined as blood pressure persistently higher than the 95th percentile for age, sex, and height for more than 3 months (4). Appropriate investigations, customised for the age, severity, history and physical examination are needed to search for an underlying cause of HT, target organ damage and to estimate the risk of complications. A thorough history and physical examination are essential so that an unusual etiology is not overlooked. HT in adolescence is usually secondary and can be caused by conditions like renal parenchymal diseases, nephrotic syndrome, renovascular diseases, drug-induced HT, tumors, coarctation of aorta, 
bronchopulmonary dysplasia or pheochromocytoma(5). Essential HT in an adolescent should be considered when there is a strong family history and other causes of secondary HT have been investigated and ruled out. Obesity is becoming increasingly common in children and adolescents. Obese children are at approximately 3 times increased risk for developing HT than non-obese children. A combination of factors including overactivity of the sympathetic nervous system (SNS), insulin resistance, and abnormalities in vascular structure and function may contribute to obesityrelated HT in children (6). SDB and OSA commonly associated with obesity in children, present with symptoms of snoring and excessive daytime sleepiness. Some studies done in adults have shown a positive association between SDB and HT $(7,8)$.

In the present case study we observed an unusual presentation of poorly controlled, persistent adolescent HT. Detailed history, physical examination and rational investigations lead to the identification of Obesity and OSA as two important risk factors in the patient. Obesity and OSA have been observed as independent risk factors for HT $(6,8)$ but there is a conflicting evidence whether OSA can lead to persistent HT in an adolescent(3). Remarkable improvement in symptoms of OSA and a better BP control was observed when the patient was titrated on continuous positive airway pressure (CPAP) supporting the view that OSA may have lead to persistent HT in this case. Mechanisms like sympathetic overactivity and hypoxia-related formation of reactive oxygen species (ROS) have been suggested to cause hypertension in OSA (9). Obesity is often associated with SDB, so weight loss by exercise and diet control certainly helped in improving symptoms of both.

CONCLUSION: This case comprehends an unusual presentation of persistent hypertension associated with sleep disordered breathing in an adolescent male. Persistent hypertension in childhood and adolescence is a diagnostic and therapeutic challenge for clinicians. A thorough history and customised investigation protocol should be used in adolescents to find out the underlying cause of hypertension and prevent further complications. Specific treatment for obstructive sleep apnea may help in relieving symptoms and achieving adequate BP control especially when no other etiology or risk factors can be identified.

\section{REFERENCES:}

1. Varda NM, Gregoric A. A diagnostic approach for the child with hypertension. Pediatr Nephrol. 2005 Apr; 20(4):499-506.

2. Sinha D, Guilleminault C. Sleep disordered breathing in children. Indian J Med Res. 2010 Feb; 131:311-20.

3. Zintzaras E, Kaditis AG. Sleep-disordered breathing and blood pressure in children: a meta analysis. Arch Pediatr Adolesc Med. 2007 Feb; 161(2):172-8.

4. National High Blood Pressure Education Program Working Group on High Blood Pressure in Children and Adolescents. The fourth report on the diagnosis, evaluation, and treatment of high blood pressure in children and adolescents. Pediatrics. 2004 Aug; 114(2 Suppl 4th Report):555-76.

5. Sumboonnanonda A, Chongcharoensuk C, Supavekin S, Pattaragarn A. Persistent hypertension in Thai children: etiologies and outcome. J Med Assoc Thai. 2006 Aug; 89 Suppl 2:S28-32.

6. Sorof J, Daniels S. Obesity hypertension in children: a problem of epidemic proportions. Hypertension. 2002 Oct; 40(4):441-7. 
7. Nieto FJ, Young TB, Lind BK, Shahar E, Samet JM, Redline S, D'Agostino RB, Newman AB, Lebowitz MD, Pickering TG. Association of sleep-disordered breathing, sleep apnea, and hypertension in a large community-based study. Sleep Heart Health Study. JAMA. 2000 Apr 12; 283(14):1829-36.

8. Grote L, Ploch T, Heitmann J, Knaack L, Penzel T, Peter JH. Sleep-related breathing disorder is an independent risk factor for systemic hypertension. Am J Respir Crit Care Med. 1999 Dec; 160(6):1875-82.

9. Buyse B, Hedner J. Sleep apnoea, hypertension and vascular disease: where are we now? Eur Respir Rev 2007; 16: 106, 169-182. 